УДК 517.4

Меджидов З.Г.

\title{
О восстановлении тензорного поля второго ранга с нулевым следом по неполным данным
}

Построен алгоритм полного восстановления тензорного поля 2-го ранга в трехмерном евклидовом пространстве по неполным интегральным данным. По линейным интегралам по прямым, пересекающим бесконечно удаленную кривую, строится соленоидальная часть поля, а по линейным интегралам от нормальной составляющей неизвестного поля с нулевым следом строится поле смещения.

Библиография: 6 названий.

An algorithm for the complete reconstruction of a tensor field of rank 2 in three-dimensional Euclidean space on incomplete integral data is constructed. The solenoid part of the field is constructed using linear integrals on straight lines that intersect a curve at infinity, and the displacement field is constructed using the traceless normal ray integrals.

Bibliography: 6 items.

Ключевые слова: лучевое преобразование, тензорное поле второго ранга, восстановление, оператор Сен-Венана, поле смещения, нормальная составляющая с нулевым следом.

Keywords: ray transform, second-rank tensor field, reconstruction, Saint-Venant operator, displacement field, traceless normal part.

\section{1. Обозначения и формулировка теоремы}

Символом $\Sigma^{m}$ обозначим линейное пространство гладких ковариантных симметричных (класса $C^{\infty}$ ) тензорных полей ранга $m, m>0$, в

(C) Меджидов 3.Г., 2020 
евклидовом пространстве $\mathbb{R}^{3}$. Определим значение поля $\Sigma^{m}$ на векторах $\xi_{1}, \xi_{2}, \ldots, \xi_{m}$ как $m$-линейную симметричную функцию $f\left(x ; \xi_{1}, \xi_{2}, \ldots, \xi_{m}\right)=f_{i_{1}, i_{2}, \ldots, i_{m}}(x) \xi_{1}, \xi_{2}, \ldots, \xi_{m}$ векторных аргументов $x \in$ $\mathbb{R}^{3}$ и $\xi_{1}=\left(\xi_{i}^{1}, \xi_{i}^{2}, \xi_{i}^{3}\right) i n \mathbb{R}^{3}, i=1,2, \ldots, m$; по повторяющемуся индексу предполагается суммирование от 1 до 3.

Лучевое преобразование поля $f \in \Sigma^{m}$ определим как совокупность интегралов вдоль прямых $l=\{x+t \theta, t \in R\}$ :

$$
\operatorname{If}(l)=\operatorname{If}(x ; \theta)=\int_{-\infty}^{\infty} f(x+t \theta, \theta, \ldots, \theta) d t=\int_{-\infty}^{\infty} f\left(x+t \theta, \theta^{m}\right) d t ;
$$

$\theta$ - направляющий вектор прямой $l$.

В данной работе будут рассматриваться пространства $\Sigma^{1}$ и $\Sigma^{2}$ полей с компактными носителями. Определим симметричный (или внутренний) дифференциал $D: \Sigma^{1} \longrightarrow \Sigma^{2}$, действующий по формуле $D \varphi=f$, $f_{i i}=\partial_{i} \varphi_{i}, f_{i j}=1 / 2\left(\partial_{i} \varphi_{j}+\partial_{j} \varphi_{i}\right), \partial_{i}=\partial /\left(\partial x^{i}\right)$ (суммирование по повторяющимся индексам не ведется).

Мы будем пользоваться также следующими обозначениями для дифференциальных операций над тензорными полями в $\mathbb{R}^{3}$, пространств и операторов в этих пространствах (см. [1-5]):

$$
\partial_{\xi} \operatorname{If}(x ; \theta)=\left\langle\xi, \nabla_{x}\right\rangle \operatorname{If}(x ; \theta), \partial_{; \xi} I f(x ; \theta)=\left.\frac{\partial}{\partial t} \operatorname{If}(x ; \theta+t \xi)\right|_{t=0} ;
$$

$\Lambda^{2}$ - пространство дифференциальных форм степени 2;

$B^{4}=\Lambda^{2} \bigotimes_{S} \Lambda^{2}-$ симметрический квадрат пространства $\Lambda^{2}$, каждый элемент $b \in B^{4}$ которого представляет собой тензорное поле с компонентами $b_{i j, k l}$, кососимметричными по индексам $(i, j)$ и $(k, l)$, а также симметричным по отношению к перестановке $(i, j) \longleftrightarrow(k, l)$;

оператор Сен-Венана $V: \Sigma^{2} \longrightarrow B^{4}$ действует на поле $f \in \Sigma^{2}$ по формуле

$$
(V f)_{i j, k l}=\partial_{i} \partial_{k} f_{j l}-\partial_{i} \partial_{l} f_{j k}-\partial_{j} \partial_{k} f_{i l}+\partial_{j} \partial_{l} f_{j k}
$$

Поля $\partial_{i}, \partial_{j}, \partial_{k}, \partial_{l}$ в последней формуле могут быть заменены на произвольные касательные векторы $\alpha, \beta, \gamma, \delta$ в $R^{3}$ :

$$
(V f)_{\alpha \beta, \gamma \delta}=\partial_{\alpha} \partial_{\gamma} f(\beta, \delta)-\partial_{\alpha} \partial_{\delta} f(\beta, \gamma)-\partial_{\beta} \partial_{\gamma} f(\alpha, \delta)+\partial_{\beta} \partial_{\delta} f(\alpha, \gamma) .
$$

Для любого потенциального поля $h=D_{\varphi}$ справедливо равенство $V h=0$, так как $V D=0$. Поэтому поле $f$ можно восстановить с точностью до потенциального слагаемого $D_{\varphi}$, где $\varphi$ - поле с компактным 
носителем. Задача восстановления соленоидальной части поля $f$ равносильно определению функции $V f([6])$, а полное восстановление поля $f$ равносильно определению полей $V f$ и $\varphi$.

В работах $[1,2]$ рассматривается преобразование вида

$$
X f(x ; \theta)=\int_{0}^{\infty} f\left(x+t \theta, \theta^{2}\right) d t
$$

определенное на семействе лучей $\{y=x+t \theta, t \geq 0\}$, где $x$ - вершина луча, $\theta$ - направляющий вектор. Решена задача восстановления поля $V f$ по данным $X f(x ; \theta)$ для лучей, вершины которых лежат на кусочногладкой кривой, удовлетворяющей условию полноты Кириллова-Туя. В статье [3] эта задача решена для тензорного поля любого ранга в пространстве произвольной размерности. В работе [1] приведено полное восстановление поля $f$ при условии, что известны дополнительные интегральные данные о поле $f$ - данные поляризационной томографии. Напомним обозначения и определения этой работы, которыми мы тоже будем пользоваться.

Пусть $f$ - тензорное поле 2-го ранга, $v$ - единичный вектор. Нормальная составляющая $f$ с нулевым следом - это тензор 2-го ранга $Q_{v} f$, определенный на плоскости $P=v^{\perp}$, ортогональной $v$, формулой

$$
\left.Q_{v} f\right|_{p}=\left.f\right|_{p}-\left.\frac{1}{2} \operatorname{tr}\left(\left.f\right|_{p}\right) i\right|_{p}
$$

где $i=\left(d u^{1}\right)^{2}+\left(d u^{2}\right)^{2}+\left(d u^{3}\right)^{2}$.

В евклидовой системе координат $\left(x_{1}, x_{2}, x_{3}\right)$, в которой $v=(0,0,1)$

$$
Q_{v} f(u, w)=\frac{1}{2}\left[\left(f_{11}-f_{22}\right) u^{1} w^{1}+f_{12}\left(u^{1} w^{2}+u^{2} w^{1}\right)+\left(f_{22}-f_{11}\right) u^{2} w^{2}\right] .
$$

Рассмотрим интегральное преобразование 2-тензора $Q_{v} f$ :

$$
T_{v} f(y ; u, w)=\int_{0}^{\infty} Q_{v} f(y+t v ; u, w) d t, \quad u, v \in P,
$$

где $v \in S^{2}, P$ - плоскость, ортогональная $v$. Ясно, что можно считать $y \in v^{\perp}=\left\{x \in R^{3}:\langle x, v\rangle=0\right\}$.

TEOPEмA 1. Пусть $\Gamma_{1}, \Gamma_{2}$ - кусочно-гладкие кривые в $R^{3}, K \subset$ $R^{3} \backslash\left(\Gamma_{1} \cup \Gamma_{2}\right)$ - компактное множество такие, что для любой точки 
$x \in K$ плоскость $H$, проходящая через $x$, пересекает каждую из кривых $\Gamma_{1}, u \Gamma_{2}$ трансверсально. Тогда тензорное поле 2-го ранга $f$ может быть восстановлено по следующим данным:

1. интегралам $X f$ вдоль лучей с вершинами $y \in \Gamma_{1}$ u вторым производным функиии $X f$ по переменной $y$;

2. интегралам $T_{v} f$ нормальной составляющей $f$ с нулевым следом и вторым производным функиии $T_{v} f$ для лучей с вершинами в точках кривой $\Gamma_{2}$.

Определим нормальное лучевое преобразование тензора $f$, отличающееся от преобразования (2) лишь пределами интегрирования:

$$
J_{v} f(y ; u, w)=\int_{-\infty}^{\infty} Q_{v} f(y+t v ; u, w) d t, \quad u, v \in P,
$$

Целью данной статьи является доказательство следующей теоремы.

TеOPEMA 2. Пусть $\Gamma_{1}, \Gamma_{2}-$ кусочно-гладкие кривые (множества направлений) на единичной сфере $S^{2}, K \subset R^{3} \backslash\left(\Gamma_{1} \cup \Gamma_{2}\right)$ - компактное множество такие, что для любой плоскости $H$, пересекающей $K$, существуют точки $M_{1} \in \Gamma_{1}, M_{2} \in \Gamma_{2}$, радиус-векторы которых параллельны H. Тогда тензорное поле 2-го ранга $f$ может быть восстановлено по следующим данным:

1. интегралам If по прямым, каждая из которых параллельна радиус-вектору какой-нибудъ точки кривой $\Gamma_{1}$;

2. интегралам $J_{v} f$ нормальной части $f$ с нулевым следом для направлений $v$, параллельных радиус-векторам точек кривой $\Gamma_{2}$.

\section{2. Доказательство теоремы}

Доказательство теоремы проведем в несколько шагов.

Шаг 1 состоит в построении поля $V f$ по данным $I f$ из пункта 1 ) теоремы 2. Эта задача решена в работе [4]. В ней доказана следующая теорема. 
TeOpema 3. Пусть $f \in S\left(R^{3}, \Sigma^{m}\right), \Gamma \subset S(n-1)-$ множество направлений, обладающее свойством: для любой гиперплоскости $H \subset R^{n}$, пересекающей носитель поля $f$, существует хотя бы одно направление $\theta \in \Gamma$, параллельное $H$. Тогда поле $V f$ может быть восстановлено по заданной функиии If $(x, \theta)$ для прямых $l=l(x, \theta), \theta \in \Gamma$.

Основное содержание доказательства этой теоремы составляет вычисление величины

$$
R_{H}\left(\xi_{1}, \omega ; \xi_{2}, \omega ; \ldots ; \xi_{m}, \omega\right)=\int_{H} V f\left(y ; \xi_{1}, \omega ; \ldots ; \xi_{m}, \omega\right) d H .
$$

Как показано в [4], для 2-тензора $f$ эту величину $R_{H}(\alpha, \omega ; \beta, \omega)$ можно вычислить по одной формулам из следующих формул в зависимости от того, какие из векторов $\alpha, \beta$ совпадают с вектором $\theta$ :

$$
\begin{gathered}
R_{H}(\theta, \omega ; \theta, \omega)=\left.\frac{1}{4} \int_{\theta^{\perp} \cap H} \frac{\partial^{2}}{\partial S^{2}} g(x+s \omega, \theta)\right|_{s=0} d x ; \\
R_{H}(\alpha, \omega ; \theta, \omega)=\left.\frac{1}{4} \int_{\theta^{\perp} \cap H} \frac{\partial}{\partial q} \frac{\partial^{2}}{\partial S^{2}} g(x+s \omega, \theta+q \alpha)\right|_{\substack{s=0 \\
q=0}} d x, \quad \alpha \neq \theta ; \\
R_{H}(\theta, \omega ; \beta, \omega)=\left.\frac{1}{4} \int_{\theta^{\perp} \cap H} \frac{\partial}{\partial q} \frac{\partial^{2}}{\partial S^{2}} g(x+s \omega, \theta+q \beta)\right|_{\substack{s=0 \\
q=0}} d x, \quad \beta \neq \theta ; \\
R_{H}(\alpha, \omega ; \beta, \omega)=\left.\frac{1}{4} \int_{\theta^{\perp} \cap H} \frac{\partial}{\partial r} \frac{\partial}{\partial q} \frac{\partial^{2}}{\partial S^{2}} g(x+s \omega, \theta+q \alpha+r \beta)\right|_{\substack{s=0 \\
q=0}} d x, \alpha \neq \theta, \beta \neq \theta ;
\end{gathered}
$$

Используя свойства симметричности и кососимметричности функции $V f$ по разным аргументам, для любых векторов $\alpha, \beta, \gamma, \delta$ можно доказать равенство

$$
\begin{aligned}
R_{H}(\alpha, \beta ; \gamma, \delta)= & R_{H}(\langle\beta \omega\rangle \alpha, \omega ;\langle\delta, \omega\rangle \gamma, \omega)-R_{H}(\langle\alpha \omega\rangle \beta, \omega ;\langle\delta, \omega\rangle \gamma, \omega) \\
& -R_{H}(\langle\beta \omega\rangle \alpha, \omega ;\langle\gamma, \omega\rangle \delta, \omega)+R_{H}(\langle\alpha \omega\rangle \beta, \omega ;\langle\gamma, \omega\rangle \delta, \omega) .
\end{aligned}
$$

В работе [5] доказана формула для вычисления $V f$, содержащая двукратное интегрирование для $m$-тензора $f, m \geq 1$. 
Шаг 2. Формула (4) дает нам преобразование Радона (покомпонентное) поля $V f$. Само поле $V f$ может быть построено применением формулы обращения этого преобразования. Полученное поле, вообще говоря, не будет иметь компактный носитель. Следующим шагом строим 2-тензор $g$ с компактным носителем такой, что $V g=V f$. Назовем его калибром поля $f$. Это поле построено в [1] как частное решение системы уравнений

$$
\begin{gathered}
\partial_{22} g_{11}-2 \partial_{12} g_{12}+\partial_{11} g_{22}=h_{33}, \\
\partial_{33} g_{11}-2 \partial_{13} g_{13}+\partial_{11} g_{33}=h_{22}, \\
-\partial_{23} g_{11}+\partial_{12} g_{13}+\partial_{13} g_{12}-\partial_{11} g_{23}=h_{23}, \\
\partial_{33} g_{22}=2 \partial_{23} g_{23}+\partial_{22} g_{33}=h_{11},
\end{gathered}
$$

где $h=V f, \partial_{i j}=\partial_{i} \partial_{j}, h_{i j}=(V f)_{k i, k j}$.

Шаг 3. Как было отмечено выше, по данным $I f(x, \theta)$ поле $f$ можно восстановить с точностью до потенциального слагаемого $D \varphi$. Третьим шагом мы приведем явные формулы для определения поля $\varphi$ по интегралам $J_{v} f$ от 2-тензора $f$, удовлетворяющего условию $V f=0$. Существование и единственность поля $\varphi$ (поля смещения) следует из следующей теоремы.

TEOPEма 4 ( [1]). Для любого тензорного поля $h$ второго ранга $c$ компактным носителем в $R^{3}$ такого, что $V h=0$, существует единственное поле смещения $\varphi$, удовлетворяющее равенству $D \varphi=h$, такое, что $\varphi=0$ в неограниченной связной компоненте $R^{3} \backslash$ suppf.

Поле смещения $\varphi$ мы построим по неполным данным, описанным в следующей теореме.

TеОрема 5. Пусть $K$ - компактное выпуклое множество в $R^{3}$, $\Gamma \subset S^{2}-$ кусочно-гладкал кривая такал, что для любой плоскости $H$, пересекапщей $K$, существует направление $v \in \Gamma$, параллельное $H$. Для любого 2-тензора $f$ с носителем в $K$, удовлетворяющего условию $V f=0$, соответствуюшее поле смещения $\varphi(D \varphi=f)$ может быть построено по интегралам $J_{v} f$ c $v \in \Gamma$.

ДокаЗАТЕЛЬСтво. Пусть $H=H(p, \omega)$ и $v \in S^{2}$ из условия теоремы. Выберем систему координат $\left(x_{1}, x_{2}, x_{3}\right)$ в $R^{3}$ так, чтобы $\omega=(1,0,0)$. Для любой прямой $L \subset H$ с направляющим вектором $v=\left(0, v^{2}, v^{3}\right)$ векторы $\omega$ и $u=\left(0, u^{1}, u^{2}\right)=v=\left(0,-v^{3}, v^{2}\right)$ образуют ортонормированный 
базис в плоскости $P$, ортогональной $v$. Величина $T_{v} f(y)$ известна для $v \in \Gamma$ и $x \in v^{\perp} \cap H$ и имеет две независимые компоненты в этом базисе:

$$
J_{v} f(y ; \omega, \omega)=\frac{1}{2} \int_{-\infty}^{\infty}\left(f_{\omega \omega}(y+t v)-f_{u u}(y+t v)\right) d t
$$

и

$$
J_{v} f(y ; \omega, u)=\int_{-\infty} \infty f_{\omega u}(y+t v) d t .
$$

Из теоремы 4 следует, что существует поле смещения $\varphi$ такое, что $D \varphi=f: f_{i i}=\partial_{i} \varphi_{i}, f_{i j}=1 / 2\left(\partial_{i} \varphi_{j}+\partial_{j} \varphi_{i}\right)$. Рассмотрим величины

$$
\begin{aligned}
& I_{1}(v, p, \omega)=\int_{v^{\perp} \cap H} J_{v} f(x ; \omega, \omega) d x, \\
& I_{2}(v, p, \omega)=\int_{v^{\perp} \cap H} J_{v} f(x ; \omega, u) d x,
\end{aligned}
$$

Выразим эти величины через компоненты $f_{i j}$ тензора $f$ и компоненты $\partial_{j} \varphi_{i}$ поля $\varphi$.

По правилу преобразования компонент ковариантного тензора

$$
\begin{gathered}
f_{\omega u}=u_{2} f_{12}+u^{3} f_{13}=\frac{1}{2} \partial_{\omega}\left(u^{2} \varphi_{2}+u^{3} \varphi_{3}\right)+\frac{1}{2}\left(u^{2} \partial_{2}+u^{3} \partial_{3}\right) \varphi_{1}, \\
\int_{-\infty}^{\infty} f_{\omega u}(x+t v) d t=\frac{1}{2} \partial_{\omega} \int_{-\infty}^{\infty}\left(-v^{2} \varphi_{2}(x+t v)+v^{2} \varphi_{3}(x+t v)\right) d t \\
+\frac{1}{2} \int_{-\infty}^{\infty}\left(-v^{3} \partial_{2}+v^{2} \partial_{3}\right) \varphi_{1}(x+t v) d t
\end{gathered}
$$

Проинтегрируем последнее равенство по переменной $x \in v^{\perp} \cap H$ и 
положим $y=x+t v$ :

$$
\begin{aligned}
I_{2}(v, p, \omega)=\frac{1}{2} \partial_{p} \int_{H}\left(-v^{2} \varphi_{2}(y)+v^{2} \varphi_{3}(y)\right) d H & \\
& +\frac{1}{2} \int_{H}\left(\left(-v^{3} \partial_{2}+v^{2} \partial_{3}\right) \varphi_{1}(y)\right) d H .
\end{aligned}
$$

Второй интеграл обращается в ноль после интегрирования по частям. Запишем производные по $v^{3}$ и $v^{2}$ от обеих частей полученного равенства:

$$
\begin{aligned}
\frac{\partial}{\partial v^{3}} I_{2}(v, p, \omega) & =-\frac{1}{2} \partial_{p} \int_{H} \varphi_{2}(y) d H \\
\frac{\partial}{\partial v^{2}} I_{2}(v, p, \omega) & =-\frac{1}{2} \partial_{p} \int_{H} \varphi_{3}(y) d H
\end{aligned}
$$

Для вычисления $I_{1}$ заметим, что по тензорному закону преобразования координат

$$
\begin{gathered}
f_{u u}=\left(u^{2}\right)^{2} f_{22}+2 u^{2} u^{3} f_{23}+\left(u^{3}\right)^{2} f_{33} \\
=\left(u^{2}\right)^{2} \partial_{2} \varphi_{2}+u^{2} u^{3}\left(\partial_{2} \varphi_{3}+\partial_{3} \varphi_{2}\right)+\left(u^{3}\right)^{2} \partial_{3} \varphi_{3} \\
=\left(v^{3}\right)^{2} \partial_{2} \varphi_{2}-v^{2} v^{3}\left(\partial_{2} \varphi_{3}+\partial_{3} \varphi_{2}\right)+\left(v_{2}\right)^{2} \partial_{3} \varphi_{3} ; \\
f_{\omega \omega}=f_{11} .
\end{gathered}
$$

Имеем

$$
\begin{aligned}
& 2 I_{1}(v, p, \omega)=\partial_{p} \int_{H}\left[\left(v^{2}\right)^{2}+\left(v^{3}\right)^{2}\right] \varphi_{1}(y) d H \\
& \quad-\int_{H}\left[\left(v^{3}\right)^{2} \partial_{2} \varphi_{2}(y)-v^{2} v^{3}\left(\partial_{2} \varphi_{3}(y)+\partial_{3} \varphi_{2}(y)\right)+\left(v^{2}\right)^{2} \partial_{3} \varphi_{3}(y)\right] d H .
\end{aligned}
$$

Второй интеграл обращается в ноль после интегрирования по частям. Поэтому

$$
\frac{\partial^{2}}{\partial\left(v^{2}\right)^{2}} I_{1}(v, p, \omega)=\frac{\partial^{2}}{\partial\left(v^{3}\right)^{2}} I_{1}(v, p, \omega)=\partial_{p} \int_{H} \varphi_{1}(y) d H
$$


Для определения компонент поля $\varphi$ воспользуемся формулой обращения преобразования Радона:

$$
\begin{gathered}
\varphi_{1}(x)=-\left.\frac{1}{8 \pi^{2}} \int_{\mathbb{S}^{2}} \frac{\partial}{\partial p} \frac{\partial^{2} I_{1}}{\partial\left(v^{2}\right)^{2}}(p, \omega)\right|_{p=\langle x, \omega\rangle} d \omega, \\
\varphi_{2}(x)=\left.\frac{1}{8 \pi^{2}} \int_{\mathbb{S}^{2}} \frac{\partial}{\partial p} \frac{\partial I_{2}}{\partial v^{3}}(p, \omega)\right|_{p=\langle x, \omega\rangle} d \omega, \\
\varphi_{3}(x)=-\left.\frac{1}{8 \pi^{2}} \int_{\mathbb{S}^{2}} \frac{\partial}{\partial p} \frac{\partial I_{2}}{\partial v^{2}}(p, \omega)\right|_{p=\langle x, \omega\rangle} d \omega,
\end{gathered}
$$

Окончание доказательства теоремы 2.

Согласно алгоритму шага 1 строим поле $V f$. Пусть $g-2$-тензорное поле с компактным носителем такое, что $V g=V f$ - калибр поля $f$ (шаг 2). Положим $h=f-g$. Для этого поля имеем $V h=V f-V g=0$. Согласно теореме 4 существует единственное поле смещения $\varphi$ такое, что $D \varphi=h$. Используя метод доказательства теоремы 5 , построим это поле по неполным данным $J h=J f-J g$ (шаг 3). Для поля $f$ имеем формулу $f=g+D \varphi$. Теорема 2 доказана.

\section{Список литературы}

[1] Palamodov V.P. On reconstruction of strain fields from tomographic data. 2015. Inverse Problems, 31:8, 85002, 12 pp.

[2] Palamodov V. P. Reconstruction of a differential form from Doppler transform. SIAM J. Math. Anal. 2009. №41. Pp. 1713-1720.

[3] Меджидов 3.Г. Обращение лучевого преобразования симметричного тензорного поля с источниками на кривой. Вестник ДГУ. Вып 6. 2013. C. 107-113.

[4] Меджидов 3.Г. Обращение лучевого преобразования тензорных полей с бесконечно удаленными источниками. Вестник ДГУ. 2015. Вып. 1. С. 19-26.

[5] Меджидов З.Г. Формулы обращения тензорной томографии по неполным данным. 2014. ДЭМИ. Вып. 2. С. 75-86 
[6] Шарафутдинов В. А. Интегральная геометрия тензорных полей. Новосибирск: Наука, 1993. - 233 с.

Меджидов З.Г. (Medzhidov Z.G.)

Дагестанский федеральный исследовательский центр РАН, Дагестанский государственный университет
Поступила в редакцию

31.08 .2020 